\title{
COVID-19 Vaccine Hesitancy in the Adult General Population of India
}

\author{
Shubhangini Sachdeva ${ }^{1}$, Sridhar Mangalesh ${ }^{2}$, Sharmila Dudani ${ }^{3}$
}

\begin{abstract}
Aim and background:Two vaccines for coronavirus disease-2019 (COVID-19), by Bharat Biotech and Serum Institute of India, have been approved by the Indian Government for administration. This study attempts to analyze vaccine hesitancy in the general adult population of India.

Materials and methods: A pan-India cross-sectional survey, adapted for GoogleForms ${ }^{\circledR}$, was circulated before the rollout of vaccines to assess vaccine hesitancy. Continuous variables are expressed as mean \pm SD and categorical variables as a percentage. The Chi-square test was used for associations.

Results: Seven hundred and sixty-two responses were analyzed. $27.2 \%$ of respondents demonstrated vaccine hesitancy. $59.4 \%$ of vaccine acceptors preferred getting the vaccine from government healthcare establishments over private-sector establishments. No significant associations were observed between vaccine acceptance and age or gender. Those who accepted the vaccine were more likely to believe that it should be compulsory $(p=0.00001)$ and safety measures should be continued even after vaccine administration $(p=0.043)$. IT-related professionals showed $82.6 \%$ vaccine acceptance, healthcare professionals displayed $73.5 \%$, while lawyers showed $53.3 \%$ acceptance. There was no association between vaccine acceptance and being a frontline worker, previously having COVID-19, or having a family member who suffered from COVID-19. Despite Delhi and Maharashtra being the highest COVID-19 burden areas, the general population of these two states was more likely to refuse the vaccine than the residents of other states $(p=0.027)$.

Conclusion: This study identified the prevalence of vaccine hesitancy in the Indian population. Consistent dialogue between the government and the public is essential for trust-building. Educational intervention through an evidenced-based approach by government authorities is the need of the hour.
\end{abstract}

Keywords: Coronavirus disease-2019, Infection, Pandemic, Vaccination refusal, Vaccine.

Journal of Medical Academics (2021): 10.5005/jp-journals-10070-0070

\section{INTRODUCTION}

Originating from Wuhan, China in December 2019, SARS-CoV-2 has been responsible for 107 million cases and 2.36 million deaths as of February 12, 2021., ${ }^{1,2}$

As of today, India has the highest number of coronavirus disease-2019 (COVID-19) cases (10.9 million), second only to the United States of America, and has seen a total of 155,000 deaths due to COVID-19. ${ }^{2}$

Though wearing masks and maintenance of social distancing are the recommended methods to halt and prevent the spread of COVID-19, India faces a unique challenge with its huge population of 136.64 crores. $^{3,4}$ Approximately $35 \%$ of the population lives in urban slums which are cramped and poorly ventilated, making social distancing practically impossible. ${ }^{5}$

Though this pandemic has affected the elderly and those with co-morbidities to a greater extent, no age-group has been spared by this disease. The clinical manifestations of COVID-19 are very protean, ranging from asymptomatic infection to fatal ARDS and multiorgan failure. Despite the availability of therapeutic options, no singular treatment is effective against SARS-COV-2. The affected may experience prolonged side effects of COVID-19, even after recovery, causing considerable morbidity.

In this scenario, vaccines offer only hope. Two vaccines (manufactured by Bharat Biotech and Serum Institute of India) have recently been approved by the Government of India for their administration. ${ }^{6}$ We attempted to study the acceptance of these vaccines in the general adult population of India. Vaccine hesitancy, if any, along with reasons for the same were also analyzed.
${ }^{1}$ Department of Medicine, Dr DY Patil Medical College, Pune, Maharashtra, India

${ }^{2}$ Department of Microbiology, Army College of Medical Sciences, New Delhi, India

${ }^{3}$ Department of Medicine, Army College of Medical Sciences and Base Hospital, New Delhi, India

Corresponding Author: Sharmila Dudani, Department of Pathology, Army College of Medical Sciences and Base Hospital, New Delhi, India, Phone: +91 9811778156, e-mail: drsdudani@hotmail.com

How to cite this article: Sachdeva S, Mangalesh S, Dudani S. COVID-19 Vaccine Hesitancy in the Adult General Population of India. J Med Acad 2021;4(1):19-23.

Source of support: Nil

Conflict of interest: None

\section{Materials and Methods}

This nationwide, cross-sectional study was conducted using a survey designed for analyzing the acceptance of the COVID-19 vaccine in the general population of India. In lieu of the ongoing pandemic, this survey was adapted for GoogleForms ${ }^{\oplus}$. The survey was circulated before the rollout of vaccines in the country, to avoid any bias.

As prior prevalence studies for COVID-19 vaccine hesitancy were unavailable, $\mathrm{P}$ was taken to be $50 \%$ to arrive at the maximum possible sample size. Precision $=4 \%, \alpha=0.05$, and a $95 \%$ confidence interval were taken and the minimum required sample size was concluded to be 601 .

(c) The Author(s). 2021 Open Access This article is distributed under the terms of the Creative Commons Attribution 4.0 International License (https://creativecommons. org/licenses/by-nc/4.0/), which permits unrestricted use, distribution, and non-commercial reproduction in any medium, provided you give appropriate credit to the original author(s) and the source, provide a link to the Creative Commons license, and indicate if changes were made. The Creative Commons Public Domain Dedication waiver (http://creativecommons.org/publicdomain/zero/1.0/) applies to the data made available in this article, unless otherwise stated. 
Demographic parameters like age, gender, education, profession, state of residence, weight, and height were recorded.

Clearance from the Institutional Ethics Committee was obtained before the circulation of the survey. Informed consent and approval of anonymized use of data for research purposes were taken from all participants. The survey consisted of 15 questions and was circulated for a duration of 3 weeks, from December 21, 2020, to January 12, 2021.

Statistical analysis was performed on the IBM statistical package for the social sciences (SPSS) for Window, Version 24.0 (Armonk, NY: IBM Corp). Continuous data are expressed as mean \pm standard deviation (SD). Categorical variables are expressed as a percentage. Categorical variables were compared using a Chi-square test and odds ratios were calculated. A $p$ value of $<0.05$ was taken to be statistically significant.

\section{Results}

The present study was conducted by analyzing the responses of 762 participants, regarding the acceptance of the COVID-19 vaccine in the general adult population of India. $72.8 \%$ (555/762) individuals responded in favor of acceptance of the vaccine while 27.2\% (207/762) demonstrated vaccine hesitancy.

The age range of the participants was from 18 to 91 years (mean age: $35.3 \pm 15.8$ ). $77.3 \%$ of respondents were in the group of $18-50$ years of age and $22.7 \%$ of respondents were in the age-group of $>50$ years. The vaccine hesitancy displayed by age-groups $18-25$,
$25-20$, and $>50$ years was $27.7,29.3$, and $23.1 \%$, sequentially. The mean age for vaccine deniers and acceptors was $34.6 \pm 15.3$ and $35.5 \pm 16.0$, respectively. There was no association between age and vaccine acceptance $(p=0.422)$.

Our study included 399 (52.4\%) males and 363 (47.6\%) females.

$36.6 \%$ of our respondents were undergraduates, $36 \%$ were postgraduates, and $27.4 \%$ of respondents had completed high school, vocational training, or were diploma holders. There was no association between education and vaccine acceptance $\left(\chi^{2}=\right.$ $0.405, p=0.817)$.

The maximum number of participants were students (31.4\%), followed by doctors and healthcare professionals (17.8\%), selfemployed professionals (12.5\%), educators (7.5\%), professionals at an MNC (6.4\%), IT-related professionals (6\%), homemakers $(5 \%)$, lawyers $(2 \%)$, and other professionals like pilots, bankers, etc. (11.4\%).

Of all respondents, $33.5 \%$ of individuals had an associated comorbid condition like hypertension, diabetes mellitus, chronic kidney disease, etc. Of these individuals, $9.2 \%$ of participants were obese.

High COVID-19 burden states such as Maharashtra and New Delhi constituted $49.5 \%$ of the total respondents.

Associations of the categorical variables with acceptance or rejection of the vaccine are shown in Table 1.

Responses to acceptable pricing of the vaccine, responses to an entity of choice for dispensing the vaccine (government vs private

Table 1: Association of vaccine acceptance with other categorical variables

\begin{tabular}{|c|c|c|c|c|c|c|c|c|}
\hline & & \multicolumn{2}{|c|}{ Acceptance of vaccine } & \multirow[b]{2}{*}{$x^{2}$} & \multirow[b]{2}{*}{ Odds ratio } & \multicolumn{2}{|c|}{$95 \%$ confidence interval } & \multirow[b]{2}{*}{$p$ value } \\
\hline & & No $(n=207)$ & Yes $(n=555)$ & & & Lower & Upper & \\
\hline \multirow{2}{*}{$\begin{array}{l}\text { Should the vaccine be } \\
\text { optional/compulsory? }\end{array}$} & Optional & $173(44.8 \%)$ & $213(55.2 \%)$ & 123.212 & 0.122 & 0.082 & 0.184 & 0.00001 \\
\hline & Compulsory & 34 (9.0\%) & $342(91.0 \%)$ & & & & & \\
\hline \multirow{2}{*}{$\begin{array}{l}\text { Should safety measures } \\
\text { be continued? }\end{array}$} & Yes & $169(25.8 \%)$ & $485(74.2 \%)$ & 4.091 & 0.642 & 0.417 & 0.989 & 0.043 \\
\hline & No & $38(35.2 \%)$ & $70(64.8 \%)$ & & & & & \\
\hline \multirow[t]{2}{*}{ State } & $\begin{array}{l}\text { Delhi and } \\
\text { Maharashtra }\end{array}$ & $116(30.8 \%)$ & $261(69.2 \%)$ & 4.898 & 1.436 & 1.042 & 1.980 & 0.027 \\
\hline & Other states & $91(23.6 \%)$ & 294 (76.4\%) & & & & & \\
\hline \multirow[t]{2}{*}{ Sex } & Female & $109(30.0 \%)$ & $254(70.0 \%)$ & 2.87 & 1.318 & 0.957 & 1.815 & 0.090 \\
\hline & Male & $98(24.6 \%)$ & 301 (75.4\%) & & & & & \\
\hline \multirow{2}{*}{$\begin{array}{l}\text { Are you a frontline } \\
\text { COVID-19 worker? }\end{array}$} & No & $188(27.4 \%)$ & $498(72.6 \%)$ & 0.200 & 1.13 & 0.656 & 1.954 & 0.655 \\
\hline & Yes & $19(25.0 \%)$ & $57(75.0 \%)$ & & & & & \\
\hline \multirow{2}{*}{$\begin{array}{l}\text { Have you ever suffered } \\
\text { from COVID-19? }\end{array}$} & No & $186(26.9 \%)$ & $505(73.1 \%)$ & 0.230 & 0.877 & 0.513 & 1.500 & 0.631 \\
\hline & Yes & $21(29.6 \%)$ & $50(70.4 \%)$ & & & & & \\
\hline \multirow{2}{*}{$\begin{array}{l}\text { Has any of your family } \\
\text { members suffered from } \\
\text { COVID-19? }\end{array}$} & No & $158(27.1 \%)$ & $426(72.9 \%)$ & 0.015 & 0.976 & 0.670 & 1.422 & 0.901 \\
\hline & Yes & 49 (27.5\%) & 129 (72.5\%) & & & & & \\
\hline \multirow{2}{*}{$\begin{array}{l}\text { Has any of your family } \\
\text { members passed away } \\
\text { due to COVID-19? }\end{array}$} & No & $178(26.8 \%)$ & 485 (73.2\%) & 0.260 & 0.865 & 0.546 & 1.371 & 0.610 \\
\hline & Yes & $29(29.3 \%)$ & 70 (70.7\%) & & & & & \\
\hline \multirow{2}{*}{$\begin{array}{l}\text { Are you aware of the } \\
\text { side effects associated } \\
\text { with a vaccine? }\end{array}$} & No & 130 (28.8\%) & 321 (71.2\%) & 1.538 & 1.231 & 0.886 & 1.709 & 0.215 \\
\hline & Yes & 77 (24.8\%) & $234(75.2 \%)$ & & & & & \\
\hline
\end{tabular}


set-up), responses to "whom should the vaccine be administered first?" and percentage-wise distribution of vaccine acceptance among various occupations are shown in Figure 1.

Reasons for vaccine hesitancy among respondents are depicted in Figure 2.

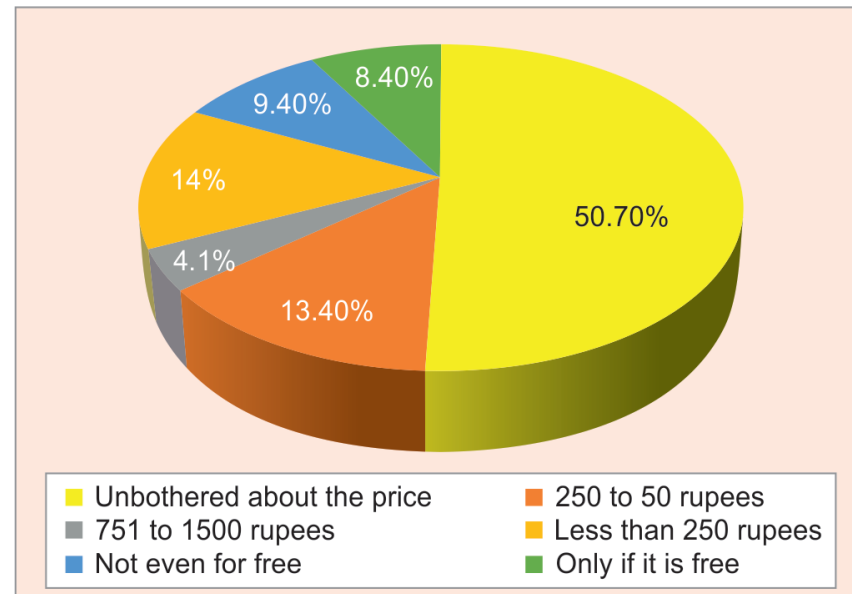

A

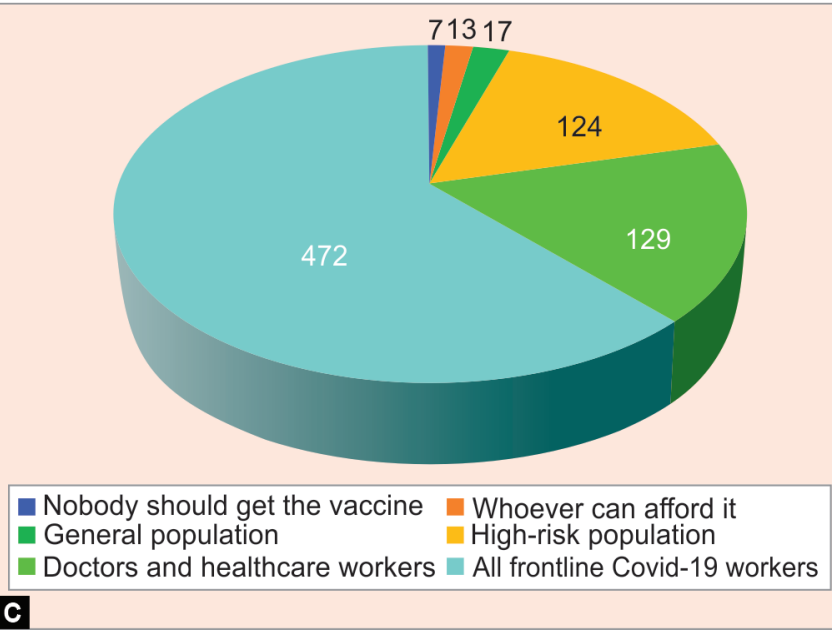

\section{Discussion}

Vaccine hesitancy is the delay in acceptance or refusal of vaccination despite the availability of vaccination services. ${ }^{7}$ In 2019, WHO identified it as one of the top 10 global threats. ${ }^{8}$

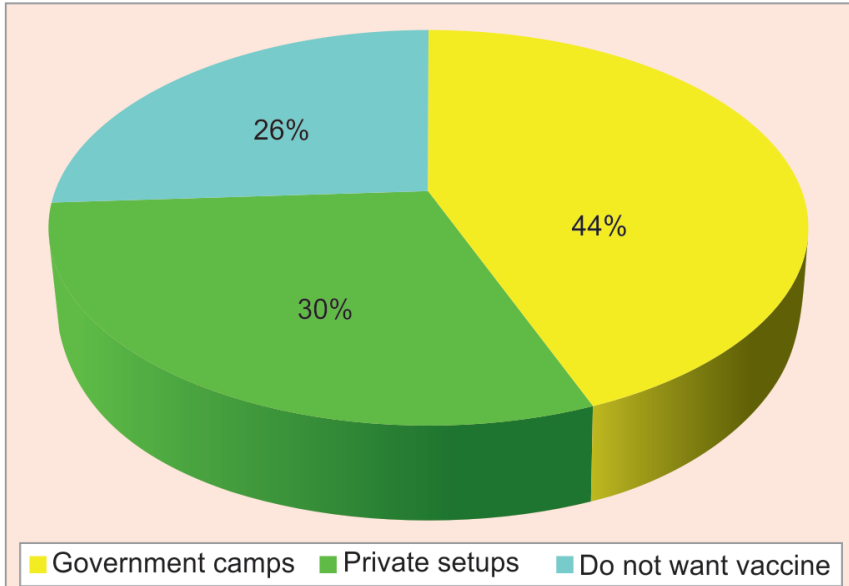

B

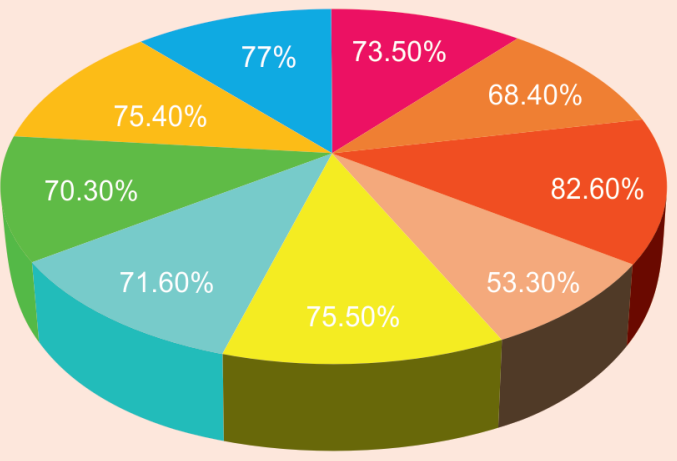

Doctors and health-related professionals

- IT-related professionals

Work at an MNC

Homemakers Self-employed Student D

Figs 1 A to D: (A) Responses to acceptable pricing of the vaccine $(n=772)$; (B) Responses to an entity of choice for dispensing the vaccine (government vs private set-up) $(n=772)$; (C) Responses to "whom should the vaccine be administered first?" $(n=772)$; (D) Percentage-wise distribution of vaccine acceptance among various occupations

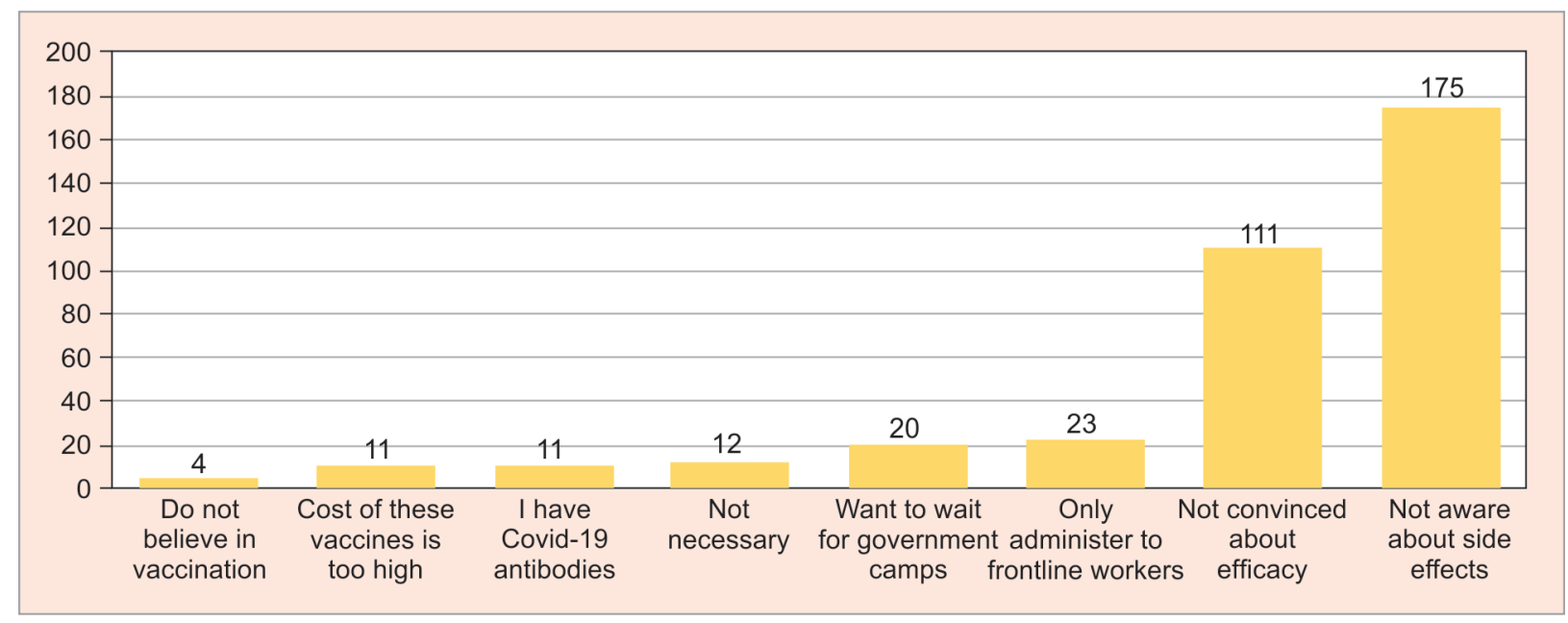

Fig. 2: Reasons for vaccine hesitancy among respondents 
This survey on acceptance of COVID-19 vaccine conducted among 762 adult participants in India, before the rollout of vaccines revealed a vaccine hesitancy of $27.2 \%$. The decision of which was not affected by age, gender, or level of education (Table 1). This was in contrast to the findings of a global survey, which reported that postgraduates and individuals above the age of 25 years were more likely to accept the vaccine when compared with those with lower levels of education and those in the group of 18 to 25 years, respectively. ${ }^{9}$ A previously conducted study showed greater vaccine acceptance among the males, reflecting a higher risk-taking behavior by this gender. ${ }^{10}$

Our study did not find any impact of being a frontline worker or prior infection with COVID-19 a family member on acceptance of the vaccine. This was in contrast with other studies where frontline workers were more likely to accept the vaccine, whereas individuals who had a family member infected with COVID-19 previously showed greater hesitancy in accepting the vaccine ${ }^{11}$ (Table 1).

Being a past COVID-19 patient or the death of an immediate family member due to COVID-19 did not significantly affect the choices of the respondent (Table 1). In another study, past COVID-19 patients and individuals whose family members had passed due to COVID-19 were equally likely to accept the vaccine as anyone else. ${ }^{11}$

The respondents were given options to choose from, regarding the potential price of the vaccine. The majority of the respondents $(50.7 \%)$ stated that they were unbothered by the price of the vaccine and $73.6 \%$ of these respondents said that they would accept the vaccine (Fig. 1A).

A majority (44\%) of respondents showed a preference in getting the vaccine from a government healthcare establishment, reflecting greater confidence in the large-scale efforts taken by the government to promote vaccine education (Fig. 1B).

Only 311 respondents were aware of the vaccine side effects, whereas 451 respondents were not (Table 1). However, no statistically significant difference was found in the acceptance or rejection of the vaccine.

The respondents were asked to choose if the vaccine should be made compulsory or optional among the general population of India. About half (49.3\%) said that the vaccine should be made compulsory, while $50.7 \%$ said that it should optional ( $p$ value: 0.00001 ) (Table 1). Those who chose to accept the vaccine were only 0.122 times as likely to assert that the vaccine is optional.

$85.8 \%$ of the participants were aware that general safety precautions against COVID-19 were to be continued even after vaccination and only $14.2 \%$ of the participants believed that precautions were unnecessary. Those who accepted the vaccine were also more likely to believe that safety measures should be continued even after the administration of the vaccine ( $p$ value: 0.043) (Table 1). This demonstrates a positive attitude of the respondents, associated with consideration for mass health and well-being.

Out of all the respondents, $61.9 \%$ agreed that all front-line COVID-19 workers be prioritized for the administration of the vaccine and only $2.2 \%$ of respondents opted for the general population to be the primary target for vaccination (Fig. 1C). It is important to highlight that many of these respondents, were a part of the general population itself.

Amidst all professions, doctors and healthcare-related professionals were not the highest acceptors of the vaccine (Fig. 1D). IT-related professionals showed $82.6 \%$ vaccine acceptance, while doctors and healthcare-related professionals displayed only $73.5 \%$ vaccine acceptance. A similar study concluded that among all professions, doctors were most likely to accept the vaccine. ${ }^{10}$

Our study concluded that despite Delhi and Maharashtra being the highest COVID-19 burden areas, the general population of these two states were more likely to refuse the vaccine than the residents of other states (OR: 1.436, $p$ value: 0.027 ) (Table 1).

No association was found between having comorbidity and acceptance of the vaccine.

The lack of awareness and knowledge of the long-term side effects of the vaccine as well as doubts regarding its efficacy were the major reasons observed for vaccine hesitancy in our study (Fig. 2). This finding was consistent with two previously conducted studies. 8,9

Vaccine hesitancy is a global public health problem. Previous research shows that approximately $60-72 \%$ herd immunity is required to effectively curb the spread of COVID-19. ${ }^{12}$ The major challenge with vaccine hesitancy is that the unimmunized population disposes the immunized population to greater risk, as well. ${ }^{13}$ A multi-layered problem requires a multifaceted approach at a group, as well as an individual level. An interpersonal interaction between trained physicians and the general population is needed to curb this hesitancy. Consistent dialogue between the government and the public is also extremely essential for trust-building. ${ }^{9}$ Educational intervention and an evidenced-based approach by government authorities are the need of the hour. Mass media also plays an imperative role in reinforcing the need for immunization. ${ }^{13}$

\section{Limitations}

This survey was conducted before the availability of the vaccines to the general population. Since the narrative is dynamic and constantly evolving, the hesitancy could be higher than portrayed in our survey. Moreover, responses might vary over time. Going ahead the availability of nasal and a single dose vaccine may impact hesitancy. Due to the lack of access to smartphones by the elderly, this study does not include a significant number of elderly individuals.

\section{References}

1. Shereen MA, Khan S, Kazmi A, et al. COVID-19 infection: Origin, transmission, and characteristics of human coronaviruses. J Adv Res 2020;24:91-98. DOI: 10.1016/j.jare.2020.03.005.

2. Worldometer. Coronavirus Information. Available from: https://www. worldometers.info/coronavirus/country/india [Accessed 12 February 2021].

3. Rahmet G, Hasanoğlu I, Aktaş F. COVID-19: prevention and control measures in community. Turkish J Med Sci 2020;50(SI-1):571-577. DOI: 10.3906/sag-2004-146.

4. Worldometer. Population Statistics. Available from: https://www. worldometers.info/world-population/india-population. [Accessed 12 February 2021].

5. The World Bank. Available from: https://data.worldbank.org/ indicator/EN.POP.SLUM.UR.ZS?locations=IN. [Accessed 12 February 2021].

6. Indian Council of Medical Research. Worldwide Covid-19 candidate vaccines. Available from: https://vaccine.icmr.org.in/covid-19vaccine. [Accessed on 1 February 2021].

7. MacDonald NE. SAGE working group on vaccine hesitancy. Vaccine hesitancy: definition, scope and determinants. Vaccine 2015;33(34):4161-4164. DOI: 10.1016/j.vaccine.2015.04.036. 
8. World Health Organisation. Ten threats to global health in 2019. Available from: https://www.who.int/news-room/spotlight/tenthreats-to-global-health-in-2019. [Accessed 12 February 2021].

9. Lazarus JV, Ratzan SC, Palayew A, et al. A global survey of potential acceptance of a COVID-19 vaccine. Nat Med 2020;2020(2):1124-1129. DOI: 10.1038/s41591-020-1124-9.

10. Grech V, Gauci C, Agius S. Vaccine hesitancy among Maltese healthcare workers toward influenza and novel COVID-19 vaccination. Early Hum Dev 2020. 105213. DOI: 10.1016/j.earlhumdev.2020. 105213.
11. Dror AA, Eisenbach N, Taiber S, et al. Vaccine hesitancy: the next challenge in the fight against COVID-19. Eur J Epidemiol 2020;35(8):775-779. DOI: 10.1007/s10654-020-00671-y.

12. Anderson RM, Vegvari $C$, Truscott J, et al. Challenges in creating herd immunity to SARS-CoV-2 infection by mass vaccination. Lancet 2020;396(10263):1614-1616. DOI: 10.1016/S0140-6736(20) 32318-7.

13. Kumar D, Noor N, Kashyap V. Vaccine hesitancy-issues and possible solutions. J Med Allied Sci 2018;8(2):55-58. DOI: 10.5455/jmas. 290153. 